\title{
Governance und Regionalentwicklung in Großschutzgebieten der Schweiz und Österreichs
}

\author{
Marco Pütz ${ }^{1}$ (D) $\cdot$ Hubert Job ${ }^{2}$
}

Eingegangen: 12. Januar 2016 / Angenommen: 17. Oktober 2016 / Online publiziert: 28. Oktober 2016

(C) Springer-Verlag Berlin Heidelberg 2016

Zusammenfassung Der Beitrag untersucht Großschutzgebiete aus der Perspektive der raumbezogenen GovernanceForschung. Im Mittelpunkt steht die Analyse der Governance-Regime ausgewählter Großschutzgebiete in Österreich und der Schweiz. Es wird eruiert, durch welche Merkmale und Strukturen die Governance-Regime der Großschutzgebiete charakterisiert sind und wie dabei die Zusammenarbeit der Akteure beurteilt wird. Der Beitrag verbessert so zum einen das Verständnis der regionalen Governance von Großschutzgebieten. Zum anderen werden mithilfe der Ergebnisse Empfehlungen für ein effizientes Management und eine bessere Organisation von Großschutzgebieten gegeben.

Schlüsselwörter Regionale Governance · GovernanceRegime · Regionalentwicklung · Großschutzgebiet · Regionaler Naturpark · Biosphärenreservat

\section{Dr. Marco Pütz}

marco.puetz@wsl.ch

Prof. Dr. Hubert Job

hubert.job@uni-wuerzburg.de

1 Wirtschafts- und Sozialwissenschaften, Eidgenössische Forschungsanstalt für Wald, Schnee und Landschaft WSL, Zürcherstrasse 111, 8903 Birmensdorf, Schweiz

2 Institut für Geographie und Geologie, Julius-Maximilians-Universität Würzburg, Am Hubland, 97074 Würzburg, Deutschland

\section{Governance and Regional Development in Protected Areas of Switzerland and Austria}

\begin{abstract}
The article investigates large protected areas from a governance research perspective. The analytical focus is on regional governance regimes of selected large protected areas in Austria and Switzerland. The analysis characterizes structures and patterns of regional governance regimes. Also, the kind and quality of cooperation between the actors is assessed. The article contributes to a deeper understanding of the regional governance of protected areas. Drawing from the empirical findings some recommendations are made for a more efficient management and organization of large protected areas.
\end{abstract}

Keywords Regional Governance - Governance Regime · Regional Development · Large Protected Area · Regional Nature Park · Biosphere Reserve

\section{Einleitung}

Der Beitrag untersucht Großschutzgebiete aus der Perspektive der raumbezogenen Governance-Forschung. Großschutzgebiete sind aus verschiedenen Gründen interessante Objekte für die Governance-Forschung. Sie sind Teil eines Mehrebenensystems, das durch Regelungsstrukturen auf verschiedenen Ebenen (supranational, national, regional, lokal) und durch Top-down- und Bottom-up-Prozesse gekennzeichnet sein kann (vertikale Koordination). Großschutzgebiete verfolgen unterschiedliche Fachziele und erfordern die Zusammenarbeit von Akteuren verschiedener Politikfelder, z. B. Natur- und Landschaftsschutz, Landund Forstwirtschaft und räumliche Planung (horizontale Koordination). Außerdem zeichnen sich Großschutzgebiete 
dadurch aus, dass staatliche (v. a. Verwaltung) und halbstaatliche sowie private Akteure (z. B. Verbände, Grundbesitzer, lokale Unternehmen, Naturtouristen) in unterschiedlichen Akteurkonstellationen auf verschiedenen räumlichen Maßstabsebenen zusammenwirken (vgl. Becken/Job 2014).

Großschutzgebiete brauchen einerseits die lokale und regionale Akzeptanz und andererseits die finanzielle und organisatorische Unterstützung auf übergeordneter, meist nationaler Ebene. Sie befinden sich damit im Spannungsfeld zwischen Selbst- und Fremdbestimmung. Besondere Brisanz erhält Letztgenannte insofern, als dass ökonomische Nachteile, die von Reservaten auf ihr Umfeld durch Nutzungsverzichte ausgehen können, oft lokal bis regional, ökologische Vorteile der Parke aber zumeist national bis global wirksam werden (Job/Mayer 2012). Weitere Friktionen können daraus resultieren, dass Großschutzgebiete in der Regel grenzüberschreitend sind und mehrere Gemeinden oder andere politisch-administrative Einheiten umfassen. Ein nächstes Spannungsfeld liegt in dem ressortübergreifenden Ansatz von Großschutzgebieten, der verschiedene Ziele verfolgt und damit unter anderem Gegenstand des Natur- und Landschaftsschutzes, der Land- und Forstwirtschaftspolitik oder der Landesplanungs- und Raumentwicklungspolitik ist. Eine häufig auftretende Konfrontationsebene entsteht zudem dadurch, dass mit der Ausweisung eines Großschutzgebietes ein neuer raumstruktureller Akteur aufscheint, der sich in bestehende Akteurkonstellationen einbringen muss. Einerseits kann er Besitzstände in Frage stellen, wodurch Raumnutzungskonflikte bedingt sein mögen, andererseits kann er neue Entwicklungen anstoßen sowie mitgestalten (Job 2010).

Diese Spannungsfelder können ganz spezifische Regelungsstrukturen und Koordinationsmuster hervorbringen, die wir im Folgenden in Anlehnung an Ostrom (1990) und Gerber, Knoepfel, Nahrath et al. (2009) als „RegionaleGovernance-Regime" bezeichnen und als zentrale Analysekategorie nutzen. Regionale-Governance-Regime entstehen durch das Zusammenspiel unterschiedlicher Mechanismen. Sie werden verstanden als die regionalspezifische Art und Weise, wie räumliche Entwicklungsprozesse gesteuert und koordiniert werden (vgl. Pütz 2004). Regionalspezifisch sind Governance-Regime deshalb, weil davon ausgegangen wird, dass es keine identischen Governance-Regime geben kann. Gleichwohl ist zu erwarten, dass bestimmte Typen von Governance-Regimen identifiziert werden können, die durch jeweils vorherrschende Merkmale charakterisiert sind. Welche Merkmale prinzipiell zur Charakterisierung von Governance-Regimen geeignet, welche GovernanceTypen analytisch zu differenzieren und wie diese in realitas anhand von konkreten Fallstudien ausgeprägt sind, wird nachfolgend ausgeführt.

Im Mittelpunkt steht dabei die Analyse der GovernanceRegime ausgewählter Großschutzgebiete in Österreich und in der Schweiz. Es wird eruiert, durch welche Merkmale und Strukturen die Governance-Regime dieser Großschutzgebiete charakterisiert sind und wie dabei die Zusammenarbeit der Akteure beurteilt wird. Der Beitrag möchte so zum einen das Verständnis der regionalen Governance von Großschutzgebieten verbessern. Zum anderen können mithilfe der Ergebnisse Schlussfolgerungen für ein effizientes Management und eine bessere Organisation von Großschutzgebieten gezogen werden, die auf die Situation in Deutschland übertragen werden könnten. Im Aufsatz werden drei Forschungsfragen bearbeitet:

- Welche regionalen Governance-Regime charakterisieren die als Fallstudien ausgewählten Großschutzgebiete?

- Inwiefern unterscheiden sich die Regionalen Governance-Regime in Naturparken und Biosphärenreservaten ${ }^{1}$ sowie in Österreich und der Schweiz?

- Wie wird die Zusammenarbeit der Akteure durch die an der Entwicklung und Organisation von Großschutzgebieten Beteiligten bewertet?

Im Anschluss an die Einleitung wird in Kap. 2 ein Überblick über die aktuelle Debatte von Governance und Großschutzgebieten gegeben. Kap. 3 bietet eine Übersicht über die Debatte um Governance in der Regionalentwicklung. Kap. 2 und 3 skizzieren jeweils den Stand der Forschung und führen in die theoretisch-konzeptionellen Grundlagen für die Analyse von Governance und Regionalentwicklung in Großschutzgebieten ein. Im Kap. 4 werden Methodik und Analyseraster für die Empirie des Beitrags vorgestellt. Kap. 5 präsentiert die Ergebnisse der empirischen Erhebungen. Abschließend werden in Kap. 6 die Resultate diskutiert und in Kap. 7 Schlussfolgerungen gezogen.

\section{Governance und Großschutzgebiete}

\subsection{Forschungsstand und theoretisch-konzeptionelle Grundlagen}

Wissenschaftliche Literatur, die sich systematisch und vergleichend mit Regionalen Governance-Regimen in Großschutzgebieten befasst, ist im deutschsprachigen Raum bis dato kaum vorhanden. Auch werden die Beiträge aus dem deutschsprachigen Raum nur in Einzelfällen an die internationale Debatte angeschlossen (z.B. Gerber/Knoepfel 2008; Hirschi 2010; Mose/Jacuniak-Suda/ Fiedler 2014). Daher zielt dieser Beitrag auf die Bearbeitung dieser beiden Defizite ab. Bisher dominieren Studien,

\footnotetext{
${ }^{1}$ Die prominentere und zugleich strengere Flächenschutzkategorie „Nationalpark“ wurde hier ausgeklammert, da sie weit weniger dem Regionalentwicklungsziel verpflichtet ist, als dies Biosphärenreservate und traditionell Naturparke sind.
} 
Abb. 1 IUCN Protected Area Matrix: Klassifikation von Großschutzgebieten nach Management-Kategorie und Governance-Typ (Borrini-Feyerabend/ Dudley/Jaeger et al. (2013: 44))

\begin{tabular}{|c|c|c|c|c|c|c|c|c|c|c|c|}
\hline \multirow{2}{*}{\begin{tabular}{|l}
$\begin{array}{r}\text { Governance } \\
\text { Type }\end{array}$ \\
Management \\
Category
\end{tabular}} & \multicolumn{3}{|c|}{$\begin{array}{l}\text { A. Governance } \\
\text { by government }\end{array}$} & \multicolumn{3}{|c|}{$\begin{array}{l}\text { B. Shared } \\
\text { governance }\end{array}$} & \multicolumn{3}{|c|}{$\begin{array}{l}\text { C. Private } \\
\text { governance }\end{array}$} & \multicolumn{2}{|c|}{$\begin{array}{l}\text { D. Governance } \\
\text { by indigenous } \\
\text { peoples and local } \\
\text { communities }\end{array}$} \\
\hline & 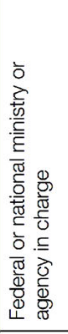 & 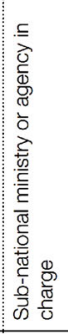 & 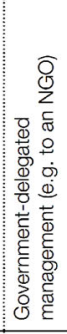 & 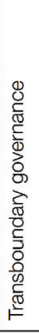 & 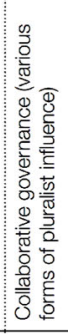 & 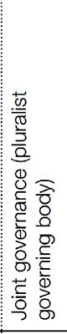 & 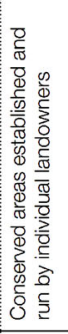 & 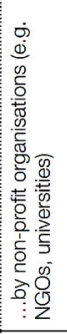 & 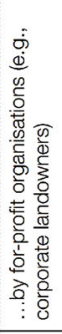 & 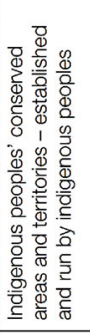 & 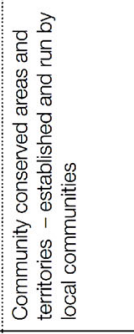 \\
\hline \multicolumn{12}{|l|}{$\begin{array}{l}\text { Ia. Strict Nature } \\
\text { Reserve }\end{array}$} \\
\hline \multicolumn{12}{|l|}{ Ib. Wilderness Area } \\
\hline \multicolumn{12}{|l|}{ II. National Park } \\
\hline \multicolumn{12}{|l|}{ III. Natural Monument } \\
\hline \multicolumn{12}{|l|}{$\begin{array}{l}\text { IV. Habitat/ Species } \\
\text { Management }\end{array}$} \\
\hline \multicolumn{12}{|l|}{$\begin{array}{l}\text { V. Protected } \\
\text { Landscape/ } \\
\text { Seascape }\end{array}$} \\
\hline $\begin{array}{l}\text { VI. Protected Area } \\
\text { with Sustainable Use } \\
\text { of Natural Resources }\end{array}$ & & & & & & & & & & & \\
\hline
\end{tabular}

die nur einzelne Fallstudien vertieft analysieren oder nur auf spezifische Aspekte von Governance fokussieren. So beruft sich Hirschi (2010) zur Untersuchung horizontaler und vertikaler Formen der Zusammenarbeit nachhaltiger Entwicklung in den Regionalen Naturparken Thal und Binntal in der Schweiz auf „Network Governance“. Gerber und Knoepfel (2008) untersuchen den Umgang mit unterschiedlichen Nutzungsansprüchen von Landschaften in Regionalen Naturpärken der Schweiz aus der Perspektive „Landscape Governance“. Görg (2007) widmet sich „Landscape Governance“ aus konzeptioneller Perspektive, ohne Empirie in Großschutzgebieten. Weiter gibt es einige Studien, die sich entweder mit Reservaten beschäftigen und Governance nur streifen, oder solche, die sich mit Regionaler Governance befassen und Großschutzgebiete nur indirekt behandeln. Lahner (2009) hat in ihrer Dissertation das „Place-Making“ durch Großschutzgebiete am Beispiel der Regionen Rhön und Schaalsee erörtert. Weixlbaumer und Mose (2006) beschäftigen sich mit dem Paradigmenwechsel von Government zu Governance anhand von Großschutzgebieten. Biosphärenreservate als ein regionales Leitinstrument wurden am Beispiel des Biosphärenparks Großes Walsertal (Österreich) von Coy und Weixlbaumer (2009) untersucht. Die Governance von kulturlandschaftlichen Handlungsräumen wurde von Gailing (2012) analysiert. Zu Regional Governance im ländlichen Raum hat Giessen (2010) gearbeitet. Gabi und Thierstein
(2005) haben sich mit Kulturlandschaften allgemein als Teil von „Metropolitan Governance“ befasst. Dahingegen leisten Moss und Gailing (2010) einen spezifischen Beitrag zu geeigneten Governance-Formen für Biosphärenreservate im Hinblick auf die drei Kategorien „sectoral interplay“, „spatial fit" und „scale“. Am Beispiel des trilateral aufgehängten, die Bundesländer Bayern, Hessen und Thüringen berührenden Biosphärenreservats Rhön diskutiert auch Pokorny (2010) Fragen der regionalen Selbststeuerung und zwar besonders unter Partizipationsgesichtspunkten (vgl. dazu auch Kraus 2015).

Auf europäischer Ebene widmen sich verschiedene Arbeiten zu ,governance of protected areas“ der Umsetzung Europäischer Naturschutzrichtlinien in den Nationalstaaten (Paavola 2004; Gibbs/While/Jonas 2007). Mehnen (2013) vergleicht die regionale Governance von Schutzgebieten der IUCN ${ }^{2}$-Kategorie V (Geschützte Landschaft) in Europa (vgl. Abb. 1). Speziell die Ausweisung neuer Schutzgebiete, als Teil der Implementation von NATURA 2000 ist ein vielfach empirisch untersuchtes Thema, beispielsweise von Grodzinska-Jurczak und Cent (2011) in Polen sowie Beunen und van Assche (2013) in den Niederlanden. Rauschmayer, van den Hove und Koetz (2009) fokussieren in ihrer Analyse von Politiken zum Erhalt der Biodiversität

${ }^{2}$ IUCN = International Union for Conservation of Nature and Natural Resources. 
auf die Rolle von Partizipation für die Governance. Parra (2010) untersucht „Multilevel Governance“ des „Parc naturel régional du Morvan“ in Frankreich. Wiederum nur indirekt Großschutzgebiete betreffend wurden von Mose, Jacuniak-Suda und Fiedler (2014) verschiedene GovernanceStile in ausgewählten LEADER ${ }^{3}$-Gebieten in Spanien, Polen und Schottland identifiziert.

Aus internationaler Sicht ist die Arbeit von Depraz (2011), der ,bonne gouvernance“ unter Akzeptanzgesichtspunkten von Großschutzgebieten diskutiert, von Bedeutung. Stoll-Kleemann und Welp (2008) benennen Faktoren für die Beeinflussung gesellschaftlicher Prozesse in Abhängigkeit unterschiedlicher Governance-Typen anhand einer groß angelegten Befragung bei Biosphärenreservaten weltweit. Dabei heben sie auf den geographischen Tatbestand $a b$, ob diese in Industrie- oder Schwellen- bzw. Entwicklungsländern gelegen sind, und debattieren Hemmnisse für die Umsetzung des sustainable development im operativen Management der Großschutzgebiete (vgl. auch Stoll-Kleemann 2010). Shafer (2015) betont die Rolle der Pufferzonen von Großschutzgebieten in diesem Kontext. Er stellt in seinem auf die IUCN-Kategorien abhebenden Beitrag die kritische Frage, ob die ,weichen' Kategorien des Gebietsschutzes, zu denen Naturparke und Biosphärenreservate gehören, dem Erhalt der Biodiversität genügend verpflichtet sind bei ihrem gleichzeitig formulierten Regionalentwicklungsauftrag. Am Beispiel von Mexiko wird diese Frage exemplifiziert durch die Studien von Brenner und de la Vega-Leinert (2014) sowie Brenner und Job (2012). Dabei geht es, mit einem speziellen Fokus auf den Tourismus, um akteurorientierte Managementformen angesichts der von der UNESCO strikt vorgegebenen Dreier-Zonierung in Kern-, Puffer-, Entwicklungszonen in Biosphärenreservaten.

\subsection{Vom Management zur Governance von Schutzgebieten}

Das Wachstum an Großschutzgebieten ist weltweit immens. Nach dem Erdgipfel von Rio 1992 sind Anzahl und Flächen von Reservaten besonders stark gestiegen (Bertzky/ Corrigan/Kemsey et al. 2012: 5 ff.): Waren es vor 25 Jahren noch elf Mio. $\mathrm{km}^{2}$, so hat sich die Schutzgebietsausdehnung auf heute weltweit über 24 Mio. $\mathrm{km}^{2}$ erhöht. Nichtsdestotrotz ist zu hinterfragen, ob das Netz an Großschutzgebieten auch tatsächlich einen essenziellen Beitrag zum eingeforderten Erhalt der biologischen Vielfalt leistet (Shafer 2015). Denn noch sind längst nicht alle Biodiversitäts-Hotspots unter Schutz gestellt. Des Weiteren existieren eine ganze Rei-

\footnotetext{
${ }^{3}$ LEADER: Förderprogramm der Europäischen Union zur Entwicklung ländlicher Räume.
}

he sogenannter ,paper parks“, die nur der besseren Statistik dienen (Stoll-Kleemann/Job 2008: 86).

Die Entwicklung des Großschutzgebiets-Managements lässt sich grob in fünf Phasen nachzeichnen (hier verkürzt übernommen nach Job/Becken/Sacher 2013; vgl. auch Plieninger/Woltering/Job 2016). Die erste Phase kann mit „Erhaltung der landschaftlichen Schönheit" betitelt werden. Sie ist von der leitenden Idee der Landschaftsästhetik gekennzeichnet. 1916 fand mit dem Gesetz über den „US National Park Service“ das nordamerikanische Totalschutzmodell seinen Anfang, welches das globale Großschutzgebietsmuster maßgeblich geformt hat. Die klassische Naturschutzkonzeption betont die Schutzwürdigkeit unberührter Natur (,preservation“). Die Auswahl der Schutzgebiete erfolgte top-down ohne Rücksicht auf die Einheimischen. Die zweite Phase lässt sich mit „Schutz einzelner Arten“ umschreiben. Dabei ging es vorrangig um den Artenschutz, wobei besonders attraktive, sogenannte emblematische Arten (z. B. Eisbär, Tiger, Berggorilla) bei der Ausweisung von Reservaten im Fokus standen. Diese tendenziell militaristische Politik ,Zäune und Strafen“ (fences and fines) beinhaltete mitunter den Ausschluss der lokalen Bevölkerung und Umsiedlung, was gleichermaßen auch für die erste Phase zutraf. Bei der dritten Phase lautete das Managementziel „Biotopschutz“. Für dieses umfassendere Biotopschutzziel war der zwischenzeitliche landschaftsökologische Erkenntnisgewinn maßgeblich, dass nämlich die Interkonnektivität und die Requisitenausstattung der Ökosysteme langfristig entscheidend sind für das Überleben und die Abundanz der charakteristischen Spezies. Auch hier waren die im Umfeld der Schutzgebiete lebenden Menschen nie wirklicher Bestandteil des Managementansatzes.

Während in den ersten 100 Jahren nach der Yellowstone-Gründung 1872 die Nationalparkanrainer weitestgehend ausgeklammert wurden, kam es danach zu einer regelrechten Zäsur im Gebietsschutz. Eine Triebfeder für die Umorientierung war das „Man and Biosphere“-Programm (MaB) (1970) der UNESCO, insbesondere mit dem Ziel, ein weltumspannendes Netz an Schutzgebieten, sogenannten Biosphärenreservaten, aufzubauen, in denen Naturschutz mit regionaler sozialer und ökonomischer Entwicklung entsprechend der sogenannten Sevilla-Strategie verknüpft werden sollte (UNESCO 1996). Als ein weiterer Meilenstein im Wandel der Schutzkonzeptionen gilt die „World Conservation Strategy“ (IUCN/UNEP/WWF 1980), die auf eine Integration von Naturschutz und Entwicklung und auf eine nachhaltige Nutzung der natürlichen Lebensgrundlagen abzielt. Diese neue Naturschutzphilosophie „Schutz und Entwicklung durch integriertes Management" steht für die vierte Phase (,conservation“), die sich ab den 1980er-Jahren besonders in Entwicklungsländern in einer Vielzahl von Projekten niederschlug, die den Schutz der Biodiversität in Reservaten mit einer loka- 
len sozioökonomischen Entwicklung zu verbinden suchen. Ziel ist es, das Management über die Schutzgebietsgrenzen hinaus zu betreiben, um damit regionalwirtschaftliche Effekte für die Einheimischen zu generieren und zwar als Kompensation für Nutzungsverzichte. Die mit dem Schutz einhergehenden Nutzungsverzichte werden durch Maßnahmen verschiedenster Art zu kompensieren versucht (z.B. verwaltungstechnisch festgelegte Ausgleichszahlungen für Wildschäden), häufig jedoch beschränkt sich die Mitwirkung auf eine schlichte Informationspflicht ohne echte Mitbestimmung der lokalen Akteure bei wichtigen Entscheidungen des Managements. Aufgrund der größeren Akteurvielfalt durch Partizipation und neue vertragliche Regeln wie beispielsweise Ausgleichszahlungen wird in dieser vierten Phase die zunehmende Bedeutung von Governance-Elementen für die Schutzgebiete deutlich, ohne dass hier bereits explizit der Begriff „,Governance“ benutzt wird.

Mit Beginn des 21. Jahrhunderts greift die fünfte Phase (,,co-management“). Es wird ein neues Konzept eingeführt, das mit „(Indigenous) Community Conservation Areas“ umschrieben werden kann (Job/Becken/Sacher 2013: 209). Es umfasst Schutzgebiete natürlicher oder kulturell modifizierter Ökosysteme mit hoher Biodiversität, die freiwillig von indigenen und lokalen Gemeinschaften entsprechend ihrer tradierten Gepflogenheiten gemanagt werden. Die betreffenden Großschutzgebiete werden von lokalen Gemeinschaften im Idealfall so gemanagt, dass der Schutz der Biodiversität mit Partizipation im engeren Sinne, also überwiegend selbstbestimmter nachhaltiger Regionalentwicklung, einhergeht. Solche Community Conservation Areas sind heute weltweit auf dem Vormarsch (Bertzky/Corrigan/ Kemsey et al. 2012). Der in der vierten Phase bereits einsetzende Wandel zu mehr Governance-Elementen, die noch eher als ,integratives“ oder „Co-Management“ bezeichnet wurden, findet in der fünften Phase seine Fortsetzung. Die Vielfalt an Akteuren, Regeln und Managementinstrumenten wird größer und Governance-Formen werden explizit als solche benannt (vgl. Borrini-Feyerabend/Dudley/Jaeger et al. 2013).

Im Jahr 2013 hat die IUCN, good governance principles“ vorgelegt, die als Aktionsplan kurz-, mittel- und langfristige Komponenten für das Management von Großschutzgebieten beinhalten. Hintergrund für diese Richtlinien ist die auf dem 5. Weltparks-Kongress in Sydney 2012 entwickelte Idee betreffend die Umsetzung des von der „Convention on Biological Diversity“ (CBD) formulierten Aichi-Ziels 11. Hierbei geht es darum, ,to help develop and implement a system for the voluntary appraisal of protected area governance quality to illuminate and communicate innovative and effective approaches to protected area governance" (Borrini-Feyerabend/Dudley/Jaeger et al. 2013: $\mathrm{XV}$ ). In diesem Kontext werden Partizipation (der betroffe- nen Akteure, besonders indigener Volksgruppen), Respekt (die Rechte und tradierte Landnutzungssysteme der Einheimischen betreffend) und ,benefit-sharing“ (Zugang zu den natürlichen Ressourcen und darauf basierende Nutzungen) als wichtigste Governance-Prinzipien genannt. Die IUCNRichtlinien differenzieren Governance anhand von drei Faktoren (Borrini-Feyerabend/Dudley/Jaeger et al. 2013: 10):

A. Schlüsselakteure: ,rightholders“vs. ,stakeholders“ (z. B. Touristen), Regierungs- (z. B. Naturparkverwaltung) vs. Nichtregierungsorganisationen (z. B. Bauernverband),

B. Machtrelationen und Machtinstrumente: unter anderem Rahmengesetze und darauf basierende Verordnungen mit Geboten bzw. Verboten, Managementplänen, Zonierungsregularien, technische Durchführungsvorgaben (z. B. zur Kulturlandschaftspflege),

C. Entscheidungsebenen: räumliche Maßstabsebenen von global bis regional/lokal, von multi- bis bilateral und die Untereinheiten eines bestehenden Schutzgebietsystems tangierend.

Anhand der jeweiligen Ausprägungen der unter A, B und $C$ genannten Parameter werden von der IUCN vier Governance-Typen für Großschutzgebiete unterschieden (BorriniFeyerabend/Dudley/Jaeger et al. 2013: 29; vgl. Abb. 1): 1) Governance by government: hierarchisch organisierte Governance durch staatliche Behörden wie beispielsweise beim Schweizerischen Nationalpark (IUCN-Kategorie Ia); 2) Shared governance: gemischte Governance, stark auf kollaboratives Management ausgerichtet, auch grenzübergreifend, z. B. Biosphärenreservat Rhön (IUCN-Kategorie V); 3) Private Governance: private Governance durch Nichtregierungsorganisationen, Unternehmen oder individuelle Rechtspersonen, z. B. wenn im Reservat ausschließlich Familieneigentum besteht, z. B. Londolozi Private Game Reserve (IUCN-Kategorie II in Südafrika; 4) Governance by indigenous peoples and local communities: Governance von indigenen Völkern und zeitweisen Großschutzgebietsbewohnern bzw. -anrainern, die mitsamt ihrem lokalen Wissen sowie traditionellen Fähigkeiten die territorialen wie auch natürlichen Ressourcen eines Gebietes kollektiv beanspruchen und als öffentliche Güter zumeist in Allmende-Form nutzen, z. B. Balanggarra in Westaustralien.

Die beiden zuletzt genannten Governance-Typen spielen in Mitteleuropa derzeit keine Rolle. Für Typ C gibt es allerdings auch in unseren Breiten bereits erste privat gemanagte Reservate, z. B. die Sielmanns Naturlandschaft Döberitzer Heide bei Berlin, ein etwa 4500 ha großes und zugleich als Naturschutzgebiet ausgewiesenes Areal. Jeder der vier Governance-Typen könnte für jede der von der IUCN unterschiedenen sechs Kategorien von Großschutzgebieten in Frage kommen (vgl. Job/Woltering/Warner et al. 2016; vgl. Abb. 1). 


\section{Governance in der Regionalentwicklung: Forschungsstand und konzeptionelle Grundlagen}

Der Governance-Begriff ist mehrdeutig, aber besser als andere Begriffe für die Untersuchung ,real existierender politischer Ordnungen“ (Mayntz 2009: 9) geeignet, weil er alle unterschiedlichen Regelungsformen umfasst (hierarchische und nicht-hierarchische, staatliche und nicht-staatliche). Der zentrale Hintergrund für die Governance-Debatte ist, dass die staatliche Handlungsfähigkeit, aber auch die Wirksamkeit und Kontrollierbarkeit des Marktes gegenwärtig grundsätzlich in Frage gestellt werden. Gleichzeitig treten neue und komplexere Themen auf, bei denen eine intensivere Zusammenarbeit verschiedener Akteurgruppen gefragt ist (Walk 2008). Governance-theoretische Ansätze gehen weiter als die politikwissenschaftliche Steuerungstheorie, weil sie anerkennen, dass die Voraussetzungen für eine zielorientierte Steuerung in der Praxis nicht erfüllt sind und die Vorstellung von einem Interventionsstaat mittlerweile überholt scheint. Die Governance-Forschung, die in der Regel interdisziplinär angelegt ist, wird auf Deutsch in einigen Übersichtsdarstellungen behandelt (z. B. von Blumenthal 2005; Schuppert 2005; Benz/Lütz/Schimank et al. 2007; Benz/Dose 2010).

Regional Governance „bezeichnet Formen der regionalen Selbststeuerung in Reaktion auf Defizite sowie als Ergänzung der marktwirtschaftlichen und der staatlichen Steuerung. Sie tritt dort auf, wo das Zusammenspiel staatlicher, kommunaler und privatwirtschaftlicher Akteure gefordert ist, um Probleme zu bearbeiten“ (Fürst 2010: 49). Der Begriff „Regional Governance“ ist in der deutschen und englischen Sprache ein Sammelbegriff für alle raumbezogenen Governance-Formen. Regional Governance bezieht sich auf die Regeln und Praktiken der Koordination räumlicher Entwicklungsprozesse. Charakteristisch für Regional Governance ist die Mitwirkung staatlicher, zivilgesellschaftlicher und privatwirtschaftlicher Akteure an Entscheidungen zu Sachverhalten im kollektiven Interesse. Damit ist Regional Governance als Begriff zwar unscharf definiert, hat sich aber mit diesem grundlegenden Verständnis in der deutschsprachigen Debatte etabliert (Fürst 2003; Pütz 2004; Diller 2005; Kleinfeld/Plamper/Huber 2006; Fürst 2010).

Neben dem Begriff „Governance“ selbst ist auch das Regionale oder der Raumbezug von Governance unscharf und der Begriff „Raum“ wenig eindeutig. Die Beiträge in Kilper (2010) betonen die wechselseitige Strukturierung und den engen Zusammenhang von Governance und Raum. Hier werden die grundlegenden Arbeiten von Benz und Dose (2010), Fürst (2003) oder Blatter und Knieling (2009) weiter gedacht und die kognitive und kommunikative Dimension bei der Konstitution oder Konstruktion von
Regionen oder Governance-Formen in den Mittelpunkt des Erkenntnisinteresses gestellt. In den letzten Jahren wurden zudem einige Arbeiten vorgelegt (meist Dissertationen), die Regional Governance empirisch untersucht haben. Es fällt auf, dass diese Studien sich oft mit spezifischen Themen der Regionalentwicklung beschäftigen oder einzelne regionalpolitische Strategien und Programme untersucht haben, unter anderem Siedlungsentwicklung (Pütz 2004), Großschutzgebiete (Fürst/Lahner/Pollermann 2005; Weber 2013; Hammer/Mose/Siegrist et al. 2016), Freiraumentwicklung und Regionalparks (Gabi/Thierstein 2005), Regionalentwicklung im ländlichen Raum wie etwa „Regionen Aktiv“ oder LEADER+ (Böcher 2008; Giessen 2010), grenzüberschreitende Regionalentwicklung (INTERREG) (Deppisch 2012; Zäch/Pütz 2014), Wirtschaftsförderung (Panebianco 2013), Raumplanung (Beutl 2010; Harrison/Growe 2014), Stadtumbau (Bernt/Haus/Robischon 2010) oder überregionale Partnerschaften (Pennekamp 2011).

Im Mittelpunkt des vorliegenden Beitrags stehen Regionalentwicklungsprozesse, die mit institutionellem Wandel auf regionaler Ebene einhergegangen sind und zur Bildung neuer Regionen geführt haben, konkret: zu Naturparken oder Biosphärenreservaten. Daher liegt es nahe, sich auf institutionalistische Ansätze zu beziehen. Konzeptionell werden im Folgenden „New Regionalism“ und „New Institutionalism“" zusammengedacht. Von Interesse für den vorliegenden Beitrag ist dabei, wie reformfähig bestehende Institutionen sind und unter welchen Bedingungen institutioneller Wandel stattfindet. Von den vier grundsätzlich zu unterscheidenden institutionalistischen Ansätzen Rational Choice, historischer, soziologischer und diskursiver Institutionalismus (vgl. Senge/Hellmann 2006) - ist der letztgenannte für den vorliegenden Beitrag besonders interessant. Der diskursive Institutionalismus fokussiert auf Kontext, Dynamik und Diskurse des institutionellen Wandels (Schmidt 2010), die hier am Beispiel regionalspezifischer Governance-Regime von Großschutzgebieten empirisch untersucht werden.

\section{Methodik}

\subsection{Datenerhebung und Datenauswertung}

Um Governance-Regime empirisch erfassen und vergleichend bewerten zu können, wurden jeweils zwei Fallstudien in Österreich und der Schweiz durchgeführt. In der Schweiz wurden der Regionale Naturpark Thal und die Biosphäre Entlebuch und in Österreich der Naturpark Zirbitzkogel-Grebenzen und der Biosphärenpark Salzburger Lungau/ Kärntner Nockberge als Fallstudien ausgewählt. Die Fallstudien sind gute Beispiele für die Entwicklung regionaler Governance-Strukturen. Als Kriterien für die Auswahl der 
Tab. 1 Vergleich ausgewählter Großschutzgebiete in Österreich und in der Schweiz

\begin{tabular}{|c|c|c|c|c|}
\hline \multirow[t]{2}{*}{ Merkmal } & \multicolumn{2}{|l|}{ Fallstudien Österreich } & \multicolumn{2}{|l|}{ Fallstudien Schweiz } \\
\hline & $\begin{array}{l}\text { Naturpark Zirbitzkogel- } \\
\text { Grebenzen }\end{array}$ & $\begin{array}{l}\text { Biosphärenreservat Lun- } \\
\text { gau/Nockberge }\end{array}$ & Naturpark Thal & $\begin{array}{l}\text { Biosphärenreservat Ent- } \\
\text { lebuch }\end{array}$ \\
\hline \multicolumn{5}{|l|}{ Raumstruktur: } \\
\hline Fläche $\left(\mathrm{km}^{2}\right)$ & 285 & 1500 & 139 & 395 \\
\hline Bevölkerung (Einwohner) & 8000 & 32.000 & 14.000 & 14.000 \\
\hline Anzahl Gemeinden & 10 & 19 & 9 & 7 \\
\hline \multicolumn{5}{|l|}{ Institutioneller Rahmen: } \\
\hline Rechtlicher Rahmen & $\begin{array}{l}\text { Landesnaturschutzgesetz } \\
\text { des Landes Steiermark }\end{array}$ & $\begin{array}{l}\text { Landesnaturschutzesetze } \\
\text { der Länder Salzburg und } \\
\text { Kärnten; Kärntner Bio- } \\
\text { sphärenpark Nockberge } \\
\text { Gesetz K-BNG }\end{array}$ & $\begin{array}{l}\text { Natur- und Heimat- } \\
\text { schutzgesetz, Pärke- } \\
\text { verordnung }\end{array}$ & $\begin{array}{l}\text { Natur- und Heimat- } \\
\text { schutzgesetz, Pärkever- } \\
\text { ordnung, Bundesinventar } \\
\text { geschützter Landschaften } \\
\text { (BLN) }\end{array}$ \\
\hline $\begin{array}{l}\text { Weitere, wichtige institu- } \\
\text { tionelle Regeln }\end{array}$ & $\begin{array}{l}\text { Teilfinanzierung durch } \\
\text { Landschaftspflegefonds } \\
\text { Steiermark; NATURA } \\
\text { 2000; Regionalversamm- } \\
\text { lung; regionale Agen- } \\
\text { da } 21 \text { für Naturpark }\end{array}$ & $\begin{array}{l}\text { Managementplan; LEA- } \\
\text { DER+; NATURA 2000; } \\
\text { Tourismus-Masterplan }\end{array}$ & $\begin{array}{l}\text { Naturparkcharta; Mana- } \\
\text { gementplan für 10 Jahre; } \\
\text { Leistungsvereinbarung } \\
\text { mit Kanton Solothurn } \\
\text { für jeweils vier Jahre; } \\
\text { jährliche Rechenschafts- } \\
\text { berichte }\end{array}$ & $\begin{array}{l}\text { Naturparkcharta; Mana- } \\
\text { gementplan für } 10 \text { Jahre; } \\
\text { Leistungsvereinbarung } \\
\text { mit Kanton Luzern für } \\
\text { jeweils vier Jahre; Natio- } \\
\text { naler Finanzausgleich; } \\
\text { Neue Regionalpolitik }\end{array}$ \\
\hline Schlüsselakteure & $\begin{array}{l}\text { Bundesland Steiermark; } \\
\text { Gemeinden; Verband } \\
\text { Naturpärke Steier- } \\
\text { mark; Tourismusver- } \\
\text { band; Großgrundbesitzer } \\
\text { (u. a. Stift St. Lambrecht, } \\
\text { Landwirte); Berg- und } \\
\text { Naturwacht Steiermark }\end{array}$ & $\begin{array}{l}\text { Regionalverband Lun- } \\
\text { gau; Großgrundbesitzer } \\
\text { (u. a. Landwirte); Bio- } \\
\text { sphärenpark-Komitee } \\
\text { (Beirat, 20 Personen); } \\
\text { Tourismusverband; Ge- } \\
\text { schäftsführer Nock-Alm- } \\
\text { Straße; Bergbahnen; } \\
\text { Schutzgemeinschaft für } \\
\text { die Entwicklung von } \\
\text { Schutzgebieten }\end{array}$ & $\begin{array}{l}\text { Region Thal (Planungs- } \\
\text { verband und Träger des } \\
\text { Naturparks); Gemeinden; } \\
\text { Landwirte; Holzhand- } \\
\text { werk }\end{array}$ & $\begin{array}{l}\text { Direktor des Biosphären- } \\
\text { reservats; Gemeindever- } \\
\text { band Entlebuch; Land- } \\
\text { wirte; Alpwirtschaftli- } \\
\text { cher Verein; Bergbahnen } \\
\text { Sörenberg }\end{array}$ \\
\hline $\begin{array}{l}\text { Spezifika der Kooperati- } \\
\text { on }\end{array}$ & $\begin{array}{l}\text { Großer Einfluss des Tou- } \\
\text { rismus; viel Aktivität } \\
\text { durch geförderte Projekte }\end{array}$ & $\begin{array}{l}\text { Themenforen; Koope- } \\
\text { ration zwischen Lungau } \\
\text { und Nockbergen, meist } \\
\text { durch Fördermaßnah- } \\
\text { men; jeweils fokussierte } \\
\text { Zusammenarbeit im Lun- } \\
\text { gau und in den Nockber- } \\
\text { gen; großer Einfluss der } \\
\text { Großgrundbesitzer (90\% } \\
\text { des Biosphärenreservats } \\
\text { sind privates Land); Bio- } \\
\text { sphärenparkbeauftragte } \\
\text { in den Gemeinden; Mit- } \\
\text { sprache der Gemeinden } \\
\text { über Regionalkonferenz }\end{array}$ & $\begin{array}{l}\text { Lange Tradition der } \\
\text { regionalen Zusammen- } \\
\text { arbeit; starke lokale Ver- } \\
\text { ankerung; Themenforen; } \\
\text { viel Aktivität durch ge- } \\
\text { förderte Projekte }\end{array}$ & $\begin{array}{l}\text { Professionelles Manage- } \\
\text { ment des Biosphären- } \\
\text { reservats; starke lokale } \\
\text { Verankerung; Partizi- } \\
\text { pation über Delegier- } \\
\text { tenversammlung mit } \\
40 \text { Personen; Themenfo- } \\
\text { ren (u. a. Landwirtschaft, } \\
\text { Energie); viel Aktivität } \\
\text { durch geförderte Projek- } \\
\text { te; großer Einfluss der } \\
\text { Landwirtschaft }\end{array}$ \\
\hline
\end{tabular}

Fallstudien wurden herangezogen: Ähnlichkeit der räumlichen Strukturen, Vergleichbarkeit des politisch-administrativen Systems (Föderalismus), kulturelle Ähnlichkeit (Sprache), Vorhandensein eines integrativen Schutzgebietsmanagements und eine erkennbare Ausrichtung auf regionale Wirtschaftsentwicklung. Außerdem entsprechen alle Fallstudien der IUCN-Kategorie V (Protected Landscape) (vgl. Abb. 1). Die untersuchten Fallstudien sind nicht repräsentativ für die Schutzgebietskategorie insgesamt oder den Governance-Typ nach IUCN und bieten lediglich interessante Einblicke in die jeweilige Governance-Praxis vor Ort.
In jeder Fallstudienregion wurden zunächst Dokumentenanalysen und anschließend halbstandardisierte, leitfadengestützte, problemzentrierte Expertengespräche durchgeführt. Für die Dokumentenanalyse wurden regionale Entwicklungskonzepte, Managementkonzepte (Charta) der Schutzgebiete, Publikationen von Verbänden und Dachorganisationen wie „Netzwerk Schweizer Pärke“, „Verband der Naturparke Österreichs“ oder „Parkforschung Schweiz“ sowie Projektbroschüren und Internetseiten berücksichtigt. Die Experteninterviews fanden in der Schweiz im Zeitraum August bis Oktober 2013 und in Österreich im Zeitraum 


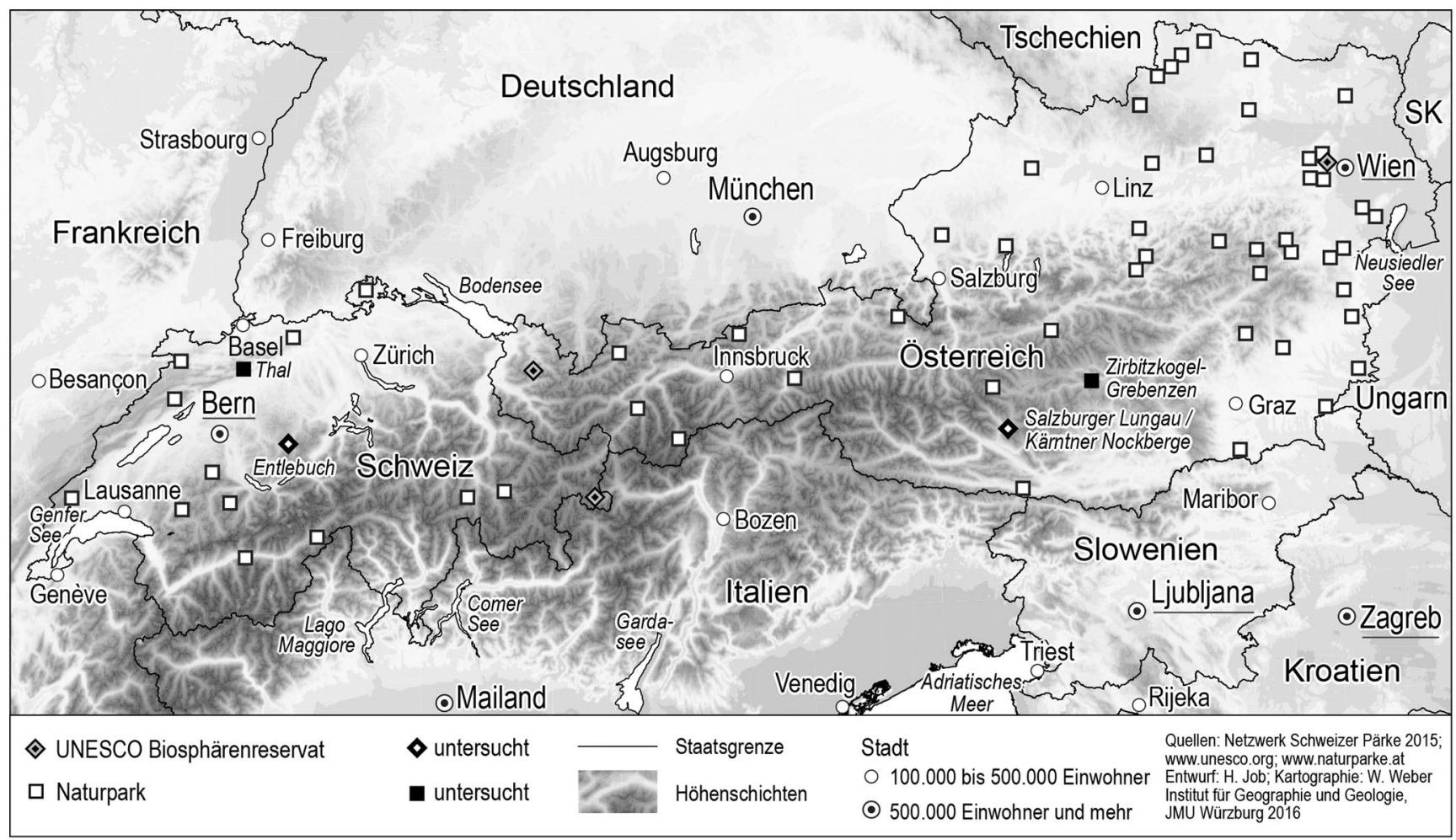

Abb. 2 Schutzgebiete in Österreich und in der Schweiz (ohne Nationalparks)

Februar bis Juni 2014 statt. Insgesamt wurden 26 Interviews geführt, davon 16 einstündige Interviews mündlich vor Ort, neun einstündige Interviews telefonisch sowie ein weiteres Interview schriftlich. Es wurden acht Interviews mit Experten außerhalb der Fallstudienregionen geführt (Externe Österreich (EA) 1-5, Externe Schweiz (ES) 1-3); je vier Interviews fanden in den beiden Naturparken statt, differenziert nach Akteuren aus der Verwaltung und Sonstigen (Naturpark Verwaltung (NV) 1-4, Naturpark Sonstige (NS) 1-4); insgesamt 10 Interviews wurden in den beiden Biosphärenreservaten durchgeführt, differenziert nach Akteuren aus der Verwaltung und Sonstigen (Biosphärenreservat Verwaltung (BV) 1-6, Biosphärenreservat Sonstige (BS) 1-4). Die Zitate wurden entsprechend dieser Codierung anonymisiert. Alle Interviews wurden transkribiert und qualitativ inhaltsanalytisch nach Mayring (2015) ausgewertet. Ziel der qualitativen Inhaltsanalyse ist es, das Interviewmaterial $\mathrm{zu}$ verdichten und eine dahinter liegende Bedeutung zu erfassen. Dazu wurde zunächst ein Kategoriensystem erstellt, das in einem ersten Schritt die vier Kategorien Raumstruktur, Institutionen, Akteure und regionale Zusammenarbeit enthielt und später weiter differenziert und sprachlich angepasst wurde. Diese Kategorien bilden sowohl die Forschungsfragen als auch die theoretisch-konzeptionellen Grundlagen des Beitrags ab und werden im nachfolgenden Kapitel als Analyseraster vorgestellt und auch zur Präsentation der Ergebnisse genutzt (vgl. Tab. 1). Danach wurden die Informationen aus den Interviews den Kategorien zugeordnet, um sie strukturieren, Häufigkeiten erkennen und die Forschungsfragen beantworten zu können. Die Inhaltsanalyse geht systematisch und theoriegeleitet vor, ist also keine freie Interpretation.

\subsection{Analyseraster}

Zur Strukturierung der Datenerhebung und schließlich zur Präsentation der Ergebnisse wird im Folgenden mit „Regionalen Governance-Regimen" gearbeitet. Wir verstehen darunter die regionalspezifische Art und Weise, wie räumliche Entwicklungsprozesse in Großschutzgebieten gesteuert und koordiniert werden. Sie entstehen durch das Zusammenspiel unterschiedlicher Mechanismen und bringen regionalspezifische Regelungsstrukturen und Koordinationsmuster hervor. Wir gehen davon aus, dass es keine identischen Governance-Regime geben kann. Gleichwohl ist zu erwarten, dass bestimmte Typen von Governance-Regimen identifiziert werden können, die durch jeweils vorherrschende Merkmale charakterisiert sind. Die wesentlichen Merkmale des Analyserasters sind (vgl. Tab. 1):

- raumstrukturelle Parameter: Fläche, Bevölkerung und Anzahl der Gemeinden,

- institutioneller Rahmen: rechtlicher Rahmen und weitere wichtige institutionelle Regeln, 
- Schlüsselakteure und

- Spezifika der Kooperation.

Das Analyseraster und unser Verständnis von Regionalen-Governance-Regimen knüpft an Ostrom (1990) und Gerber, Knoepfel, Nahrath et al. (2009) an. Das „Institutional Analysis and Development Framework" von Ostrom (1990) (vgl. auch Ostrom 2009; Clement 2010; McGinnis 2011) ist speziell für die Analyse regionaler Governance interessant, weil hier explizit die lokal- und regionalspezifischen Attribute berücksichtigt werden, die gerade auch Großschutzgebiete stark prägen.

\section{Ergebnisse}

\subsection{Rahmenbedingungen für die regionale Governance von Großschutzgebieten in Österreich und der Schweiz}

\subsection{1 Österreich}

In Österreich fällt Naturschutz in Gesetzgebung und Vollzug in die Kompetenz der Bundesländer. Es existiert auf der übergeordneten Ebene der Republik kein Rahmengesetz wie in Deutschland, was die Zusammenarbeit über Bundesländergrenzen erschwert. Deshalb besteht z. B. der Nationalpark Hohe Tauern aus formaljuristisch drei separaten Teilgebieten der Länder Kärnten, Salzburg und Tirol (mit drei Verwaltungsstellen, drei Direktoren, drei Haushalten). Die Bundesländer sind unter anderem für die Errichtung und Aufhebung von Großschutzgebieten zuständig. Die Gemeinden kompensieren Schutzmaßnahmen von privaten Grundbesitzern über sogenannte Vertragsnaturschutzmodelle. Bei der Zulassung einer Schutzmaßnahme durch den Besitzer des betroffenen Gebietes erfolgt eine direkte Ausgleichszahlung durch das betreffende Bundesland. Solche Verträge können ähnlich einem Pachtvertrag wieder aufgehoben werden. Zahlreiche Maßnahmen in den Großschutzgebiets-Gemeinden werden von Vereinen getragen, z. B. das Projekt „Netzwerk-Land“, das darauf abzielt, ländliche Regionen und damit auch Großschutzgebiete zu vernetzen. Je nach Schutzgebietskategorie sind vor allem die jeweiligen Naturschutzabteilungen der einzelnen Bundesländer an der Entwicklung und Organisation der Großschutzgebiete beteiligt. Auch die Fauna-Flora-Habitat-Richtlinie (FFH) der Europäischen Union (EU) spielt in Österreich eine wichtige Rolle. Sie ist Grundlage für den Aufbau des europaweiten Schutzgebietsnetzes „NATURA 2000“ und muss in jedem Bundesland in Landesrecht und in vielen Landesgesetzen umgesetzt werden. Für den Naturschutz setzen sich in Österreich Natur- und Landschaftsschutzorganisationen, wie z. B. der „Naturschutzbund“ ein.
Der Einfluss der privaten Grundbesitzer, Bauern, Jäger und anderer auf die Schutzgebiete ist groß. Sie organisieren sich in sogenannten Schutzgemeinschaften, um sich gegen naturschutzfachliche Fremdbestimmung zu wehren und eigene Landnutzungsinteressen durchzusetzen. Ein Beispiel ist die „Schutzgemeinschaft der Grundbesitzer in Kärnten“, welche die Anliegen der Grundbesitzer bei der Ausweisung von Schutzgebieten vertritt.

Derzeit existieren in Österreich 48 Naturparke, die zwischen 20.000 und 70.000 ha groß sind und insgesamt eine Fläche von rund 500.000 ha umfassen (vgl. Abb. 2). Die ältesten davon datieren aus dem Jahr 1979 (und sind damit etwa 20 bzw. 10 Jahre jünger als die französischen oder deutschen Vorbilder). Naturparke werden in Österreich als geschützte Landschaften, die vom Menschen durch schonende Landnutzung und Landschaftspflegemaßnahmen erhalten werden, definiert und sollen Schutz, Erholung, Bildung und Regionalentwicklung gleichrangig erfüllen (Verband der Naturparke Österreichs 2016: 4 f.). Diese traditionell gewachsenen, historischen Kulturlandschaften werden als Vertreter charakteristischer österreichischer Landschaftstypen angesehen. Nur bei Zustimmung aller betroffenen Gemeinden wird das naturschützerische Prädikat „Naturpark" verliehen.

Drei von der UNESCO anerkannte Biosphärenreservate, sogenannte Biosphärenparks, gibt es momentan in Österreich: Großes Walsertal (19.200 ha), Wienerwald (105.645 ha) und Salzburger Lungau/Kärntner Nockberge (149.000 ha). Auch sie verfolgen nicht zuletzt explizite Regionalentwicklungsziele im Sinne des Sustainable-development-Paradigmas und versuchen, diese unter ihrem sehr ambitionierten, weil inhaltlich viel umfassenderen Naturschutzetikett als dies bei den Kategorien Natur- oder Nationalpark der Fall ist, in der Öffentlichkeit zu kommunizieren, z. B. mittels eines Kochbuchs zu regionalen Gerichten aus Biosphärenreservaten (Köck/Umhack/Diry 2013). Zwei Gebiete, Untere Lobau und Neusiedler See, die in Teilen die Nationalparks Donauauen und Neusiedler See umfassten, wurden 2016 von der UNESCO-Liste genommen. Zudem wurden bereits 2014 zwei weitere vormalige Biosphärenreservate - der Gurgler Kamm in den Ötztaler Alpen und der hochalpine Gossenköllesee - von der UNESCO-Liste zurückgezogen, da sie viel zu klein waren und insbesondere nicht dem Sevilla-Standard entsprachen.

\subsubsection{Schweiz}

In der Schweiz setzen das Natur- und Heimatschutzgesetz sowie die Pärkeverordnung (PäV) den gesetzlichen Rahmen für Großschutzgebiete (Schweizerischer Bundesrat 2007). Laut Schweizer Pärkeverordnung sind das Regionen, die über seltene und vielfältige Lebensräume verfügen, eine Landschaft mit besonderer Schönheit und Eigenart darstel- 
len sowie eine geringe Beeinträchtigung dieser besonderen Werte zeigen. Sind diese Voraussetzungen erfüllt, so kann mithilfe der Unterstützung der Bevölkerung und mit der Organisation einer Pärketrägerschaft die Ausgestaltung und Entwicklung der Region in Angriff genommen werden. In Regionalen Naturpärken soll eine nachhaltige ökonomische und gesellschaftliche Entwicklung über einen naturnahen Tourismus oder auch die Förderung und Entwicklung von regionalen Produkten erreicht werden. Ein explizites Ziel der Naturpärke ist die Erhaltung und Aufwertung der Natur und Landschaft.

Über einen Parkvertrag ist der Ein- und Austritt von Gemeinden in die Trägerschaft geregelt. Wenn sich der Parkperimeter verändert, ist das Bundesamt für Umwelt verpflichtet, den räumlichen Zuschnitt des Großschutzgebietes neu zu prüfen. Für die Aufhebung eines ganzen Großschutzgebietes ist jedoch das Bundesamt für Umwelt verantwortlich. Mit der sogenannten „Park Charta“ muss jeder Regionale Naturpark ein Managementkonzept vorlegen, das mindestens zehn Jahre Bestand hat und damit über die üblichen Legislaturperioden der Politik hinausreicht. ${ }^{4}$ Ebenfalls nach zehn Jahren müssen alle Großschutzgebiete entscheiden, ob sie weiter bestehen möchten. Zur Finanzierung von Großschutzgebieten ist der vertikale Finanzausgleich zwischen Bund und Kantonen wichtig. So kann der Bund strategisch und die Kantone können operativ steuern.

Auf Bundesebene ist in der Schweiz das Bundesamt für Umwelt für Großschutzgebiete zuständig. Es prüft die Anträge zur Anerkennung als Großschutzgebiet und zur Benutzung des entsprechenden Etiketts und gewährt Finanzhilfen. Dem Bundesamt für Umwelt stehen dafür jährlich 20 Mio. Franken für die Förderung der Großschutzgebiete zur Verfügung. Das Bundesamt für Umwelt hat dabei eine Aufsichtsfunktion über die korrekte Verwendung des Park- und Produkte-Labels. Des Weiteren hat das Amt eine Beratungs- und Coaching-Funktion für Kantone und die Großschutzgebiete. Ebenfalls auf übergeordneter Ebene koordiniert das „Netzwerk Schweizer Pärke“ den Erfahrungsaustausch und die Öffentlichkeitsarbeit aller Schweizer Großschutzgebiete und vertritt ihre Interessen international. Die „Parkforschung Schweiz“ unterstützt und begleitet die Großschutzgebiete bei der Forschung und deren Zusammenarbeit bei übergeordneten Themen. Von den zivilgesellschaftlichen Akteuren ist die Natur- und Landschaftsschutzorganisation „Pro Natura“ wichtig.

Derzeit sind in der Schweiz insgesamt 20 Großschutzgebiete in Betrieb oder werden zu errichten versucht (Weissen 2009; vgl. Abb. 2); diese belegen insgesamt 650.083 ha. 15 davon sind Regionale Naturpärke, deren Größe zwi-

\footnotetext{
${ }^{4}$ Damit ist man dem Vorbild der französischen „Parc Naturels Régionaux“ gefolgt, deren Geschichte bis ins Jahr 1970 zurückreicht (Job 1993).
}

schen ungefähr 14.000 und 125.000 ha variiert (Netzwerk Schweizer Pärke 2015: 5). Regionale Naturpärke entstanden in der Schweiz erst seit $2008^{5}$ (noch einmal 30 Jahre später als in Österreich). Mehrere Projekte für neue Großschutzgebiete sind inzwischen wieder von der Bildfläche verschwunden, z. B. 2014 der Regionale Naturpark Neckertal, weil die dortige Bevölkerung starke Einschränkungen fürchtete. Aufgrund ähnlicher Befürchtungen hat sich 2015 eine Gemeinde der Biosfera Val Müstair - Parc Naziunal (19.900 ha; erweitert den bereits 1914 gegründeten Schweizerischen Nationalpark in Richtung des benachbarten italienischen Nationalparks Stilfser Joch) gegen eine Erweiterung ausgesprochen - mit der Folge, dass dieses Biosphärenreservat von der UNESCO hinsichtlich des internationalen Status kritisch unter die Lupe genommen wird. Die Biosphäre Entlebuch (39.400 ha) ist das zweite von der UNESCO anerkannte Biosphärenreservat und gleichzeitig ein Regionaler Naturpark von nationaler Bedeutung.

\subsection{Governance in ausgewählten Großschutzgebieten}

\subsubsection{Regionaler Naturpark Thal}

Die Region Thal (Schweiz) ist durch ihre geographische Abgeschlossenheit und eine traditionell gute regionale $\mathrm{Zu}$ sammenarbeit charakterisiert. Das Regionale-GovernanceRegime des Naturpark Thal wird stark durch die seit 1969 bestehende regionale Planungsorganisation ,Region Thal ' geprägt, die auch die Trägerschaft für den Regionalen Naturpark Thal wahrnimmt. Das Beispiel zeigt, dass eine etablierte, funktionierende Zusammenarbeit auf regionaler Ebene, organisiert durch einen regionalen Entwicklungsträger eine schnelle und erfolgreiche Errichtung von Großschutzgebieten unterstützen kann. Außerdem wurde hier von Anfang an die Landwirtschaft eingebunden: „Die Landwirtschaft haben wir sehr früh involviert. Das war überhaupt [...] die allererste Akteursgruppe, die wir einbezogen haben beim Entwickeln der Parkidee und wir haben ihr auch aufgezeigt, dass keine Einschränkungen damit verbunden sind" (Interview NV 1). Interessant am Beispiel des Regionalen Naturpark Thal ist, dass nach der Errichtung des Naturparks Thal 2010 die Zusammenarbeit der wirtschaftlichen, zivilgesellschaftlichen und politischen Akteure noch gesteigert werden konnte. Großschutzgebiete können also dazu beitragen, etablierte Strukturen der Zusammenarbeit weiter zu vertiefen und zu intensivieren.

Für die Planung, Realisierung und Finanzierung von Projekten sind die Programmvereinbarungen zwischen Kanton und Park sowie zwischen Kanton und Bund wichtig und

\footnotetext{
5 Der Schweizerische Nationalpark aus dem Jahr 1914 wird hier wegen seines besonderen Status (für ihn gibt es ein eigenes Bundesgesetz) nicht mitgerechnet.
} 
damit ein zentrales Element des Regionalen GovernanceRegimes. Von den Interviewpartnern wird die Zusammenarbeit zwischen den Bereichen Landwirtschaft, Gastronomie und Parkverwaltung als gut bewertet. Es werden jedoch auch Potenziale bei Gastronomie und Landwirtschaft gesehen, die Zusammenarbeit zu verbessern und vor allem die Kooperationsbereitschaft zu steigern. Kritisch gesehen wird die Konkurrenz zum in unmittelbarer Nähe gelegenen, ebenfalls neuen Regionalen Naturpark Jurapark Aargau. Eine Zusammenlegung wäre aus organisatorischer und finanzieller Sicht effektiver, scheint aber aufgrund von kulturellen Differenzen derzeit nicht möglich. Die Akzeptanz des Großschutzgebietes hat nach Meinung der Interviewpartner nach der Einrichtung etwas abgenommen, ist jedoch immer noch auf hohem Niveau.

\subsubsection{Naturpark Zirbitzkogel-Grebenzen}

Der österreichische Naturpark Zirbitzkogel-Grebenzen, gegründet 1983, verfolgt das ehrgeizige Ziel, finanziell unabhängig von Fördermaßnahmen des Bundes zu werden. Charakteristisch für das Regionale-Governance-Regime dieses Großschutzgebiets ist die intensive Kooperation mit dem Tourismusverband, da die Naturparkverwaltung mit Touristikern besetzt ist. Eine intensive Zusammenarbeit aller Akteure findet vor allem zu Anfang einer neuen EU-Förderperiode statt. Daraus kann geschlossen werden, dass die in Aussicht stehenden finanziellen Mittel als Anreiz für die Zusammenarbeit von Akteuren sehr förderlich sein können. Eine Vielzahl an Projekten und Initiativen weist auf eine gute Vernetzung der Akteure hin. So gibt es eine eigene Naturparkzeitschrift, einen Naturleseweg und Bestrebungen, Angebote zur Burnout-Prävention zusammen mit anderen Partnern aufzubauen. Die Akzeptanz wird generell als gut angesehen, scheint aber in den Bereichen Jugend und Landwirtschaft ausbaufähig. Projekte, die nicht in der Region selbst entstanden sind, werden kritisch gesehen: „Das ist dann auch schief gelaufen, weil es nicht in der Region entstanden ist. Das ist von oben draufgesetzt worden. Da waren irgendwelche Burschen irgendwo in der Welt. Ich glaube in Frankreich und in der Schweiz haben sie so etwas gesehen und dann sind überall in der ganzen Steiermark so Leitprojekte in schwächere Regionen hingesetzt worden“ (Interview NS 2).

\subsubsection{Biosphäre Entlebuch}

Die Biosphäre Entlebuch verkörpert seit 2008 den ersten Regionalen Naturpark von nationaler Bedeutung in der Schweiz. Bereits seit 2001 ist das Entlebuch UNESCOBiosphärenreservat, das einzige nach den Sevilla-Kriterien der UNESCO von 1996. Eine Besonderheit hinsichtlich des Regionalen Governance-Regimes ist die intensive
Einbindung von zivilgesellschaftlichen Akteuren durch verschiedene Themenforen. Wichtig für die Finanzierung des Biosphärenreservats sind die kantonalen Einnahmen aus dem nationalen Finanzausgleich, weil das Entlebuch eine strukturschwache Region ist. Wichtig für die gute Zusammenarbeit zwischen den wirtschaftlichen Akteuren ist die Rolle der Landwirtschaft. Das zeigt sich zum einen an der Vermarktung von regionalen Produkten auch über die Grenzen des Biosphärenreservats hinaus. Zum anderen wird das an der nach wie vor hohen Bedeutung der Landwirtschaft für die regionale Identität deutlich: ,wir haben ja drei, vier Prozent noch aktive Bevölkerung in der Landwirtschaft, aber im Kopf haben wir immer noch dreißig, vierzig Prozent Bauern“ (Interview BV 2). Am Beispiel vom Biosphärenreservat Entlebuch wird auch deutlich, dass ein Regionales Governance-Regime nicht zwingend eine rechtliche Grundlage braucht, damit Akteure zusammenarbeiten und Projekte verwirklichen. Denn diese befindet sich derzeit noch in der Erarbeitungsphase.

\subsubsection{Biosphärenpark Salzburger Lungau/Kärntner Nockberge}

Der die Landesgrenzen übergreifende Biosphärenpark Lungau/Nockberge (Österreich) besteht erst seit dem Jahr 2012 und hat zwei Verwaltungen. Dadurch ist das RegionaleGovernance-Regime durch verschiedene administrative und organisatorische Strukturen im selben Biosphärenreservat geprägt, was die Transaktionskosten erhöht. Die Intensität der Zusammenarbeit ist in den Kärntner Nockbergen weiter entwickelt als im Lungauer Teil, weil die Kärntner Nockberge vor der Ausweisung als Biosphärenreservat eine Zeit lang Nationalpark waren. ${ }^{6}$ Dadurch konnten bereits Verwaltungs- und Vernetzungsstrukturen wie beispielsweise Themenforen übernommen werden, die im Lungauer Teil erst aufgebaut werden mussten. Es haben sich jedoch schnell Bürgervereine organisiert, die ein großes Interesse an der Entwicklung des Biosphärenreservats zeigen.

In den Expertengesprächen wurde deutlich, dass die $\mathrm{Zu}$ sammenarbeit zwischen beiden Teilen des Biosphärenreservates lediglich in der Anfangsphase ausgeprägt war und danach zurückgegangen ist. Als hinderlich für die Zusammenarbeit wurden die Dominanz der Landesregierungen bei Großschutzgebieten und der damit verbundene doppelte gesetzliche Rahmen durch zwei Naturschutzgesetze angesehen. In den Kärntner Nockbergen ist die Akzeptanz insbesondere von Seiten der Großgrundbesitzer reserviert.

\footnotetext{
${ }^{6}$ Da dieser allerdings nicht den internationalen IUCN-Standards entsprochen hat, wurde er deklassifiziert, insbesondere weil von nationalstaatlicher Ebene Druck ausgeübt wurde, indem die in Österreich bei Nationalparken übliche hälftige Etatisierung durch die Republik unterblieb.
} 
Generell kann festgestellt werden: „dass sich die Region durch den ganzen Partizipationsprozess und die Erstellung eines Leitbildes über alle Bereiche das erste Mal mit dem Thema Regionalentwicklung als Ganzes auseinandergesetzt hat" (Interview BS 3).

\section{Diskussion der Ergebnisse}

Die wesentlichen Merkmale der untersuchten Großschutzgebiete sind in Tab. 1 zusammengefasst. Neben den raumstrukturellen Merkmalen Fläche, Bevölkerung und Anzahl der Gemeinden stehen hier die in Kap. 1 eingeführten Merkmale für Regionale-Governance-Regime im Mittelpunkt: institutioneller Rahmen (rechtlicher Rahmen, weitere wichtige institutionelle Regeln), Schlüsselakteure sowie Spezifika der Kooperation.

Österreich und die Schweiz unterscheiden sich hinsichtlich des rechtlichen Rahmens und der politischen Zuständigkeit für Großschutzgebiete. Während in Österreich die Bundesländer zuständig und Schutzgebiete durch Landesgesetze geregelt sind, ist dies in der Schweiz beim Bund angesiedelt (vgl. Tab. 1). Wie die unterschiedliche gesetzliche und politische Verankerung zu bewerten ist oder welche Folgen damit verbunden sein könnten, ist nicht Gegenstand dieses Beitrags. Es wäre aber interessant, das in zukünftigen Studien zu untersuchen. Wichtig ist, dass die Governance von Schutzgebieten immer als Multilevel Governance zu verstehen ist und das konkrete lokale Schutzgebietsmanagement vor Ort immer in übergeordnete rechtliche Strukturen und politische Verantwortlichkeiten eingebettet ist. Neben der lokalen räumlichen Situation und den historischen Entwicklungspfaden spielen vor allem die gesetzlichen Grundlagen eine zentrale Rolle für die Errichtung von Großschutzgebieten. So ermöglichte in der Schweiz erst die Revision des Natur- und Heimatschutzgesetzes von 2007 neue Regionale Naturpärke oder andere Großschutzgebiete von nationaler Bedeutung. Die Gesetzesrevision war unter anderem notwendig, damit die Schweiz das internationale Übereinkommen zur biologischen Vielfalt (Secretariat of the Convention on Biological Diversity 2011) umsetzen kann.

In den Interviews wurde deutlich, dass Naturraum und Lage sowie Geschichte und bisherige Erfahrungen in der Regionalentwicklung einen großen Einfluss auf die Qualität und Intensität der regionalen Zusammenarbeit haben. Als Beispiel sei hier die Region Thal (Schweiz) genannt, deren gute regionale Zusammenarbeit stark durch die geographische Abgeschlossenheit der Talschaft und den Aufbau professioneller Regionalentwicklungsstrukturen im Laufe der letzten Jahrzehnte geprägt ist - und dies bereits vor der Existenz des Großschutzgebietes war. Ähnlich ist die Situation im Naturpark Zirbitzkogel-Grebenzen (Österreich).
Hier wird deutlich, dass positive gemeinsame Erfahrungen in der Zusammenarbeit essenziell für eine schnelle und erfolgreiche Errichtung von Naturparken sind. Mit der Errichtung des Regionalen Naturparkes Thal konnte nach Aussage aller Interviewpartner die regionale Zusammenarbeit der Akteure weiter gesteigert werden.

Für die Entstehung der Großschutzgebiete sind einzelne Akteure als Promotoren wichtig, die das Projekt „Großschutzgebiet" engagiert vorantreiben (vgl. u.a. Böcher 2008). Dies liegt zum Teil daran, dass vor der Errichtung der untersuchten Großschutzgebiete die Strukturen zur Zusammenarbeit gefehlt und Akteure nur schwach vernetzt waren. Die Ausweisung von Großschutzgebieten ist in wirtschaftlich schwachen Regionen erfolgreicher und wird durch Handlungsdruck erleichtert (Job/Kraus/Merlin et al. 2013). Eine wesentliche Gemeinsamkeit ist die Bildung von Themenforen oder Arbeitsgruppen in den Großschutzgebieten, bei welchen sich insbesondere Bürger, Vereine und Verbände an der Entwicklung von Projekten für das Großschutzgebiet beteiligen können.

Hinsichtlich der Machtverhältnisse ist anzumerken, dass keines der untersuchten Großschutzgebiete finanziell autark ist. Alle Großschutzgebiete sind auf Förderinstrumente von außen angewiesen. Nahezu alle finanziellen Förderungen werden von übergeordneter staatlicher Ebene angeboten, die damit eine enorme Machtposition einnimmt, auch wenn gleichermaßen eine Bottom-up-Strategie zur Durchführung von Projekten vor Ort und für die Akzeptanz unerlässlich ist.

Wichtig für die Einrichtung und weitere Entwicklung der Großschutzgebiete ist, dass alle untersuchten Gebiete nicht mit dem primären Ziel des Naturschutzes entstanden sind, sondern durch einen gemeinsamen regionalpolitischen Handlungsbedarf, die wirtschaftlichen Verhältnisse im ländlichen Raum zu verbessern. In Bezug auf die Governance-Strukturen hieße das im Prinzip, dass hier nicht nur staatliche, sondern auch privatwirtschaftliche Akteure stärker präsent sein müssten. Aktuell kann das jedoch noch nicht festgestellt werden. Nach wie vor dominieren in den untersuchten Fällen klar die hoheitlichen Akteure, die auch vor Einrichtung des Großschutzgebietes die Regionalentwicklung geprägt haben. Inwiefern sich langfristig die Akteurzusammensetzung noch verändern und Großschutzgebiete damit das Potenzial für Entrepreneurship im ländlichen Raum (vgl. Baumgartner/Pütz/Seidl 2013) verbessern können, wird die Zukunft zeigen.

Die vier Fallstudien können den Governance-Typen nach IUCN zugeordnet werden (vgl. Abb. 1) und weisen Elemente von Governance-Typ A „Governance by government" und Governance-Typ B ,Shared governance“ auf. Auf den ersten Blick entsprechen alle vier Fallstudien dem Typ A und hier dem Subtyp „Sub-national ministry or agency in charge“, weil jedes Mal eine regionale Schutz- 
gebietsverwaltung vor Ort federführend ist, jeweils mit starkem Einfluss und Rahmensetzung von übergeordneter Ebene: Bund für die Schweizer Schutzgebiete, Bundesland für die Österreichischen Schutzgebiete. Auf den zweiten Blick wird durch die Empirie deutlich, dass alle vier Fallstudien auch wichtige Elemente von Typ B aufweisen. So ist der bundesländerübergreifende Biosphärenpark Salzburger Lungau/Kärntner Nockberge ein Beispiel für „transboundary governance“. Außerdem sind die große Bedeutung der kommunalen Verankerung, der interkommunalen Zusammenarbeit in Gemeindeverbänden und der Einbezug vieler nichtstaatlicher Akteure (z. B. Landwirte, Tourismusverbände) starke Hinweise für ,collaborative governance“" oder, ,joint governance“, also Subtypen von B. Diese schwierige Zuordnung von nur vier, noch dazu ähnlichen Schutzgebieten zu nur einem Governance-Typ weist darauf hin, dass die Typen A und B doch recht schematisch sind. Nicht nur in den untersuchten Schutzgebieten in einem föderalen Kontext, sondern generell ist zu erwarten, dass alle Schutzgebiete durch Multilevel Governance und durch vielfältige Formen der Zusammenarbeit von Ebenen, Sektoren und Akteuren geprägt sind. Daher ist kritisch zu fragen, ob es im IUCN-Schema einen eigenen Typ B „Shared governance“ überhaupt braucht.

\section{Schlussfolgerungen}

Der Beitrag hat das Verständnis der regionalen Governance von Großschutzgebieten durch zwei Befunde pointiert. Erstens konnte gezeigt werden, dass Großschutzgebiete - wie in föderalen Systemen üblich - zwar Teil einer Multilevel Governance sind, aber der Einfluss der überregionalen und nationalstaatlichen Ebene sehr groß ist. Dieser Einfluss zeigt sich zum einen im Engagement von Natur- und Umweltschutzinitiativen, die zwar lokal aktiv sind, ihre Aktivität aber stark national koordinieren. Zum anderen erweist sich dieser Einfluss in der Bedeutung der rechtlichen Voraussetzungen zur Einrichtung der Großschutzgebiete sowie vor allem bei der finanziellen und organisatorischen Unterstützung ihrer Maßnahmen in den Regionen, z. B. beim Aufbau leistungsfähiger Geschäftsstellen oder bei der Kopplung finanzieller Unterstützung an neue Initiativen. Daraus lässt sich folgern, dass die Gemeindeautonomie quasi die Funktion eines Vetos erhält, um Großschutzgebiete dieses Typs zu verhindern.

Um Großschutzgebiete einrichten und erfolgreich betreiben zu können, Qualitätsstandards zu sichern, ihr Potenzial als Katalysator für regionale Entwicklungsprozesse nutzen und damit letztlich auch regionale Handlungsfähigkeit einschließlich strategischer Entscheidungen und Budget-Hoheit herstellen zu können, sind dagegen überregionale Strukturen und die direkte Unterstützung des Bundes bzw. der Republik und indirekt auch der EU hinsichtlich ihrer Strukturförderprogramme sehr wichtig. Für die Zukunft sind damit Koordination, Erfahrungsaustausch und strategische Interessensvertretung von Großschutzgebieten auf überregionaler Ebene wie beispielsweise im „Netzwerk Schweizer Pärke“ von zentraler Bedeutung. Um die nationale Koordination von Großschutzgebieten in Deutschland $\mathrm{zu}$ verbessern, ist zu überlegen, ein spezielles Bundesprogramm oder eine neue Gemeinschaftsaufgabe Großschutzgebiete zu formulieren, um die Reservate institutionell stärker zu fördern und somit auch eine Qualitätssicherung von Seiten des Bundes betreiben zu können. Denn wenn die naturschützerischen Prädikate inhaltsleer werden, werden sie auch bedeutungslos in ihrer Vorbildfunktion für die ,Normallandschaft" ohne Label.

Zweitens deuten die in den Großschutzgebieten existierenden Spannungsfelder zwischen den einzelnen Sektoren und den administrativen Ebenen darauf hin, dass der segregativ funktionierende Flächenschutz häufig alles andere als ein einfacher Weg ist, um Naturschutz- oder Biodiversitätsziele zu erreichen. Denn die damit angestrebte integrierte ländliche Entwicklung wird vor Ort vielfach so interpretiert, dass wirtschaftliche Ziele der Regionalentwicklung dominieren und die Integration von Wachstums- und Ausgleichs- sowie Schutzzielen nur ansatzweise und lediglich auf kleinen Flächen gelingt. Klar ist aber, es braucht den Flächenschutz unbedingt, da der integrative Naturschutz außerhalb von Großschutzgebieten noch stärker den liberalen Marktgesetzen und damit einer Intensivierung der Flächennutzung unterliegt und häufig viel zu kleinflächig daherkommt. Es ist daher zu diskutieren, ob eventuell die Governance von Großschutzgebieten so gestaltet werden sollte, dass sie mit eigenen Verantwortlichkeiten und Budgets klarer von anderen Sektoralpolitiken (Landwirtschaft, Tourismus) getrennt wird. Hinzu kommt, dass sich die potenziell für die sektorübergreifende Koordination von raumwirksamer Aktivität wichtige Raumplanung bei Großschutzgebieten in der Regel eher zurückhält, weil sie sich mehr um das Siedlungsgebiet kümmert und vorrangig für die Entwicklung von Siedlungs- und Verkehrsflächen interessiert. Die räumliche Planung fühlt sich derzeit stark ökonomischen Interessen der Raumentwicklung verpflichtet, denkt immer weniger strategisch und betreibt kaum eine langfristige Flächenhaushaltspolitik außerhalb des besiedelten Raumes (vgl. ARL 1999).

\section{Literatur}

ARL - Akademie für Raumforschung und Landesplanung (Hrsg.) (1999): Flächenhaushaltspolitik. Feststellungen und Empfehlungen für eine zukunftsfähige Raum- und Siedlungsentwicklung. Hannover. = Forschungs- und Sitzungsberichte der ARL 208.

Baumgartner, D.; Pütz, M.; Seidl, I. (2013): What Kind of Entrepreneurship Drives Regional Development in European Non-core 
Regions? A Literature Review on Empirical Entrepreneurship Research. In: European Planning Studies 21, 8, 1095-1127.

Becken, S.; Job, H. (2014): Protected Areas in an Era of Global-Local Change. In: Journal of Sustainable Tourism 22, 4, 507-527.

Benz, A.; Dose, N. (Hrsg.) (2010): Governance - Regieren in komplexen Regelsystemen. Eine Einführung. Wiesbaden.

Benz, A.; Lütz, S.; Schimank, U.; Simonis, G. (Hrsg.) (2007): Handbuch Governance - Theoretische Grundlagen und empirische Anwendungsfelder. Eine Einführung. Wiesbaden.

Bernt, M.; Haus, M.; Robischon, T. (Hrsg.) (2010): Stadtumbau komplex: Governance, Planung, Prozess. Darmstadt.

Bertzky, B.; Corrigan, C.; Kemsey, J.; Kenney, S.; Ravilious, C.; Besançon, C.; Burgess, N. (2012): Protected Planet Report 2012: Tracking Progress Towards Global Targets for Protected Areas. Gland, Cambridge.

Beunen, R.; van Assche, K. (2013): Contested delineations: planning, law, and the governance of protected areas. In: Environment and Planning A 45, 6, 1285-1301.

Beutl, H. (2010): Regional Governance und Regionalplanung. Zwei Fallbeispiele aus Niederösterreich. Wien. = Abhandlungen zur Geographie und Regionalforschung 12.

Blatter, J.; Knieling, J. (2009): Metropolitan Governance - Institutionelle Strategien, Dilemmas und Variationsmöglichkeiten für die Steuerung von Metropolregionen. Hannover, 224-269. = Forschungs- und Sitzungsberichte der ARL 231.

Böcher, M. (2008): Regional Governance and Rural Development in Germany: the Implementation of LEADER+. In: Sociologia Ruralis 48, 4, 372-388.

Borrini-Feyerabend, G.; Dudley, N.; Jaeger, T.; Lassen, B.; Pathak Broome, N.; Phillips, A.; Sandwith, T. (2013): Governance of Protected Areas: From understanding to action. Gland. = Best Practice Protected Area Guidelines Series 20.

Brenner, L.; de la Vega-Leinert, A. C. (2014): La gobernanza participativa de áreas naturales protegidas. El caso de la Reserva de la Biosfera El Vizcaíno. In: Région y Sociedad 26, 59, 184-213.

Brenner, L.; Job, H. (2012): Challenges to Actor-Oriented Environmental Governance: Examples from Three Mexican Biosphere Reserves. In: Tijdschrift voor Economische en Sociale Geografie $103,1,1-19$

Clement, F. (2010): Analysing decentralised natural resource governance: proposition for a "politicised" institutional analysis and development framework. In: Policy Sciences 43, 2, 129-156.

Coy, M.; Weixlbaumer, N. (Hrsg.) (2009): Der Biosphärenpark als regionales Leitinstrument. Das Große Walsertal im Spiegel der Nutzer. Innsbruck. $=$ Alpine space - man \& environment 10 .

Deppisch, S. (2012): Governance Processes in Euregios. Evidence from Six Cases across the Austrian-German Border. In: Planning Practice and Research 27, 3, 315-332.

Depraz, S. (2011): Les territoires de nature protégée, de la théorie participative aux pratiques de bonne gouvernance. In: Bulletin de 1'Association de géographes français $88,4,365-374$.

Diller, C. (2005): Regional Governance by and with Government. Die Rolle staatlicher Rahmensetzungen und Akteure in drei Prozessen der Regionsbildung. Habilitationsschrift. Technische Universität Berlin.

Fürst, D. (2003): Steuerung auf regionaler Ebene versus Regional Governance. In: Informationen zur Raumentwicklung 8/9, 441-450.

Fürst, D. (2010): Regional Governance. In: Benz, A.; Dose, N. (Hrsg.): Governance - Regieren in komplexen Regelsystemen. Eine Einführung. Wiesbaden, 49-68.

Fürst, D.; Lahner, M.; Pollermann, K. (2005): Regional Governance bei Gemeinschaftsgütern des Ressourcenschutzes: das Beispiel Biosphärenreservate. In: Raumforschung und Raumordnung 63, $5,330-339$

Gabi, S.; Thierstein, A. (2005): Der „Regionalpark“ als Handlungsfeld der Metropolitan Governance. In: Raumforschung und Raumordnung $63,1,21-31$.
Gailing, L. (2012): Sektorale Institutionensysteme und die Governance kulturlandschaftlicher Handlungsräume. In: Raumforschung und Raumordnung 70, 2, 147-160.

Gerber, J.-D.; Knoepfel, P. (2008): Towards integrated governance of landscape development: The swiss model of regional nature parks. In: Mountain Research and Development 28, 2, 110-115.

Gerber, J.-D.; Knoepfel, P.; Nahrath, S.; Varone, F. (2009): Institutional Resource Regimes: Towards sustainability through the combination of property-rights theory and policy analysis. In: Ecological Economics 68, 3, 798-809.

Gibbs, D.; While, A.; Jonas, A. E.G. (2007): Governing nature conservation: the European Union Habitats Directive and conflict around estuary management. In: Environment and Planning A 39, $2,339-358$.

Giessen, L. (2010): Regional Governance für ländliche Räume - innovativer Ansatz, politischer Gegenwind und der Weg vorwärts. In: Raumforschung und Raumordnung 68, 1, 3-14.

Görg, C. (2007): Landscape governance. The "politics of scale" and the "natural" conditions of places. In: Geoforum 38, 5, 954-966.

Grodzinska-Jurczak, M.; Cent, J. (2011): Expansion of nature conservation areas: problems with Natura 2000 implementation in Poland? In: Environmental Management 47, 1, 11-27.

Hammer, T.; Mose, I.; Siegrist, D.; Weixlbaumer, N. (Hrsg.) (2016): Parks of the Future. Protected Areas in Europe Challenging Regional and Global Change. München.

Harrison, J.; Growe, A. (2014): From places to flows? Planning for the new 'regional world' in Germany. In: European Urban and Regional Studies 21, 1, 21-41.

Hirschi, C. (2010): Strengthening Regional Cohesion: Collaborative Networks and Sustainable Development in Swiss Rural Areas. In: Ecology and Society 15, 4, 16.

IUCN - International Union for Conservation of Nature and Natural Resources; UNEP - United Nations Environment Programme; WWF - World Wildlife Fund (1980): World Conservation Strategy. Gland.

Job, H. (1993): Naturparks in Frankreich. „Parcs naturels régionaux “ und Erfahrungen für deutsche Naturparke. In: Naturschutz und Landschaftsplanung 25, 3, 105-111

Job, H. (2010): Welche Nationalparke braucht Deutschland? In: Raumforschung und Raumordnung 68, 2, 75-89.

Job, H.; Becken, S.; Sacher, P. (2013): Wie viel Natur darf sein? Schutzgebietskonzepte im Wandel der Zeit. In: Standort 37, 4, 204-210.

Job, H.; Kraus, F.; Merlin, C.; Woltering, M. (2013): Wirtschaftliche Effekte des Tourismus in Biosphärenreservaten Deutschlands. Bonn-Bad Godesberg. = Naturschutz und Biologische Vielfalt 134.

Job, H.; Mayer, M. (2012): Forstwirtschaft versus Waldnaturschutz: Regionalwirtschaftliche Opportunitätskosten des Nationalparks Bayerischer Wald. In: Allgemeine Forst- und Jagdzeitschrift 183, $7 / 8,129-144$.

Job, H.; Woltering, M.; Warner, B.; Heiland, S.; Jedicke, E.; Meyer, P.; Nienaber, B.; Plieninger, T.; Pütz, M.; Rannow, S.; von Ruschkowski, E. (2016): Biodiversität und nachhaltige Landnutzung in Großschutzgebieten. In: Raumforschung und Raumordnung 74, 6. doi:10.1007/s13147-016-0440-5.

Kilper, H. (Hrsg.) (2010): Governance und Raum. Baden-Baden.

Kleinfeld, R.; Plamper, H.; Huber, A. (Hrsg.) (2006): Regional Governance. Steuerung, Koordination und Kommunikation in regionalen Netzwerken als neue Formen des Regierens. Göttingen.

Köck, G.; Umhack, M.; Diry, C. (2013): The Austrian Biosphere Reserves. A (connoisseur's) world beyond the cookery book. In: eco.mont 5, 2, 43-47.

Kraus, F. (2015): Nachhaltige Entwicklung in Biosphärenreservaten: Regionale Wertschöpfungsketten diskutiert am Beispiel der Dachmarke Rhön. Würzburg. = Würzburger Geographische Arbeiten 114. 
Lahner, M. (2009): Regional Governance in Biosphärenreservaten. Eine Analyse am Beispiel der Regionen Rhön und Schaalsee unter Einbeziehung von Place-Making. Stuttgart.

Mayntz, R. (2009): Einleitung. In: Mayntz, R. (Hrsg.): Über Governance. Institutionen und Prozesse politischer Regelung. Frankfurt am Main, 7-12. = Schriften aus dem Max-Planck-Institut für Gesellschaftsforschung 62 .

Mayring, P. (2015): Qualitative Inhaltsanalyse. Grundlagen und Techniken. Weinheim/Basel.

McGinnis, M. D. (2011): An introduction to IAD and the language of the Ostrom workshop: a simple guide to a complex framework. In: Policy Studies Journal 39, 1, 169-183.

Mehnen, N. (2013): Protected landscapes - the great hope of European area protection policy? A comparative study of governance in IUCN category $\mathrm{V}$ areas. Groningen.

Mose, I.; Jacuniak-Suda, M.; Fiedler, G. (2014): Regional GovernanceStile in Europa. Eine vergleichende Analyse von Steuerungsstilen ausgewählter LEADER-Netzwerke in Extremadura (Spanien), Warmińsko-Mazurskie (Polen) und Western Isles (Schottland). In: Raumforschung und Raumordnung 72, 1, 3-20.

Moss, T.; Gailing, L. (2010): Institutionelle Herausforderungen und Governance-Formen für nachhaltige Entwicklung von Biosphärenreservaten. In: Deutscher Rat für Landespflege (Hrsg.): Biosphärenreservate sind mehr als Schutzgebiete. Wege in eine nachhaltige Zukunft. Meckenheim, 123-125. = Schriftenreihe des Deutschen Rates für Landespflege 83.

Netzwerk Schweizer Pärke (2015): Panorama Schweizer Pärke 2015. Bern.

Ostrom, E. (1990): Governing the commons: The evolution of institutions for collective action. New York.

Ostrom, E. (2009): A General Framework for Analyzing Sustainability of Social-Ecological Systems. In: Science 325, 5939, 419-422.

Paavola, J. (2004): Protected areas governance and justice: theory and the European Union's Habitats Directive. In: Environmental Sciences 1, 1, 59-77.

Panebianco, S. (2013): Standortfaktor Regional Governance auf dem Prüfstand. Theoretische Überlegungen und empirische Analysen zur Bedeutung regionaler Steuerungssysteme für die Wirtschaftsentwicklung von Regionen. Hamburg.

Parra, C. (2010): Sustainability and multi-level governance of territories classified as protected areas in France: the Morvan regional park case. In: Journal of Environmental Planning and Management 53, 4, 491-509.

Pennekamp, S. (2011): Überregionale Partnerschaften - was können sie leisten? In: Raumforschung und Raumordnung 69, 4, $245-256$

Plieninger, T.; Woltering, M.; Job, H. (2016): Implementierung des Ökosystemleistungs-Ansatzes in deutschen Biosphärenreservaten. In: Raumforschung und Raumordnung 74, 6. doi:10.1007/ s13147-016-0438-z.

Pokorny, D. (2010): Erfahrungen und Perspektiven zur Regionalen Selbststeuerung (Regional Governance) aus dem Biosphärenreservat Rhön. In: Deutscher Rat für Landespflege (Hrsg.): Biosphärenreservate sind mehr als Schutzgebiete. Wege in eine nachhaltige Zukunft. Meckenheim, 127-136. = Schriftenreihe des Deutschen Rates für Landespflege 83.

Pütz, M. (2004): Regional Governance - Theoretisch-konzeptionelle Grundlagen und eine Analyse nachhaltiger Siedlungsentwicklung in der Metropolregion München. München.
Rauschmayer, F.; van den Hove, S.; Koetz, T. (2009): Participation in EU biodiversity governance: how far beyond rhetoric? In: Environment and Planning C 27, 1, 42-58.

Schmidt, V. A. (2010): Taking ideas and discourse seriously: explaining change through discursive institutionalism as the fourth 'new institutionalism'. In: European Political Science Review 2, $1,1-25$.

Schuppert, G. F. (Hrsg.) (2005): Governance-Forschung. Vergewisserung über Stand und Entwicklungslinien. Baden-Baden.

Schweizerischer Bundesrat (2007): Verordnung über die Pärke von nationaler Bedeutung (Pärkeverordnung, PäV) vom 7. November 2007, SR 451.36.

Secretariat of the Convention on Biological Diversity (2011): Nagoya Protocol on Access to Genetic Resources and the Fair and Equitable Sharing of Benefits Arising from their Utilization to the Convention on Biological Diversity. Montréal.

Senge, K.; Hellmann, K.-U. (Hrsg.) (2006): Einführung in den NeoInstitutionalismus. Wiesbaden.

Shafer, C. L. (2015): Cautionary Thoughts on IUCN Protected Area Management Categories V-VI. In: Global Ecology and Conservation 3, 331-348.

Stoll-Kleemann, S. (2010): Faktoren für die Beeinflussung gesellschaftlicher Prozesse in Biosphärenreservaten. In: Deutscher Rat für Landespflege (Hrsg.): Biosphärenreservate sind mehr als Schutzgebiete. Wege in eine nachhaltige Zukunft. Meckenheim, 116-119. = Schriftenreihe des Deutschen Rates für Landespflege 83.

Stoll-Kleemann, S.; Job, H. (2008): The Relevance of Effective Protected Areas for Biodiversity Conservation: An Introduction. In: Gaia 17, S1, 86-89.

Stoll-Kleemann, S.; Welp, M. (2008): Participatory and Integrated Management of Biosphere Reserves: Lessons from Case Studies and a Global Survey. In: Gaia 17, S1, 161-168.

UNESCO - United Nations Educational, Scientific and Cultural Organization (1996): Biosphere Reserves. Seville Strategy and the Statutory Framework of the World Network. Paris.

Verband der Naturparke Österreichs (2016): Österreichische Naturparke - Landschaften voller Leben. Graz.

von Blumenthal, J. (2005): Governance - eine kritische Zwischenbilanz. In: Zeitschrift für Politikwissenschaft 15, 4, 1149-1180.

Walk, H. (2008): Partizipative Governance: Beteiligungsformen und Beteiligungsrechte im Mehrebenensystem der Klimapolitik. Wiesbaden.

Weber, F. (2013): Naturparke als Manager einer nachhaltigen Regionalentwicklung. Probleme, Potenziale und Lösungsansätze. Wiesbaden.

Weissen, A. (2009): The park creation boom in Switzerland. In: eco.mont 1, 2, 67-68.

Weixlbaumer, N.; Mose, I. (2006): Protected Areas as a Tool for Regional Development? In: Siegrist, D.; Clivaz, C.; Hunziker, M.; Iten, S. (Hrsg.): Exploring the Nature of Management. Rapperswil, 149-154.

Zäch, C.; Pütz, M. (2014): Regional Governance in der grenzüberschreitenden Zusammenarbeit. Eine Analyse des INTERREGProgramms „Alpenrhein-Bodensee-Hochrhein“. In: disP - The Planning Review 50, 4, 29-42. 